\title{
Predictive Model for the Diagnosis of Tuberculous Pleural Effusion
}

\author{
Denise Duprat Neves ${ }^{1}$, Ricardo Marques Dias ${ }^{1}$ and Antônio José Ledo A. da Cunha ${ }^{2}$ \\ ${ }^{1}$ Federal University of the State of Rio de Janeiro (UNIRIO); ${ }^{2}$ Federal University of Rio de Janeiro (UFRJ); Rio de Janeiro, RJ, Brazil
}

\begin{abstract}
This study developed a predictive model to identify pleural tuberculosis. A consecutive cases study of patients investigating the cause of pleural effusion, in an area of high prevalence of tuberculosis (Rio de Janeiro, Brazil). Clinical and laboratory variables were compared among patients with tuberculosis (TB) and without tuberculosis (NTB), individually and using logistic regression. The performance was described as diagnostic accuracy, compared to a gold standard in a masked way. We have studied 104 TB patients, 41 with malignant, 29 transudates, 28 parapneumonic, 13 with miscellaneous diseases. After identification of individual discrimination power aided by clinical, radiological and laboratory variables, the following ones were included in a multivariate analysis: ADA, total leukocytes, percentile of lymphocytes, protein, lactate dehydrogenase, duration of disease, age and gender. A logistic regression model to predict pleural tuberculosis including the five first variables showed the best performance. A receiver operating characteristic curve identified the best cutoff at 0.7 , resulting in a sensitivity and specificity of more then $95 \%$. The predictive model improved the specificity of ADA alone, keeping its sensitivity. This model seems helpful when a microbiological or histological diagnosis of pleural tuberculosis could not be established. External validation of these results is necessary before recommendation for routine application.

Key-Words: Sensitivity and specificity, tuberculosis, pleural effusions, predictive model, diagnosis.
\end{abstract}

Pleural tuberculosis (TB) incidence is generally related to the local prevalence of diseases. In Brazil were TB have a high prevalence (principally in Rio de Janeiro), this is the major cause of pleural effusions, responsible for almost half of all diagnosis [1,2].

The gold standard for the tuberculosis diagnosis is the identification of the bacillus, especially through culture of mycobacterium, which is time consuming and not sensitive enough. Cultures are positive only in one third of pleural fluid samples and around two third of pleural biopsy specimens $[3,4]$. Therefore, the presence of caseating granuloma on pleural biopsy specimen is accepted as diagnosis. In high prevalence areas of tuberculosis, the presence of noncaseating granuloma, associated with other characteristics, is also acceptable as pleural tuberculosis diagnosis. This procedure increases the sensitivity at the expense of lowering specificity. The diagnosis of pleural tuberculosis has been greatly improved by the use of biochemical markers, which are faster and can be more sensitive. The pleural fluid activity of adenosine deaminase (ADA) is one of the best [1,2,5,6-8], providing reliable basis for a treatment decision, particularly in excluding the diagnosis of tuberculosis, due to its high sensitivity, independent of HIV serologic status [2,9]. On the other hand, the test specificity does not provide enough precision to confirm the diagnosis, because ADA increases (false-positive results) in many other conditions like: empyema fluid, rheumatoid disease and lymphoproliferative disorders $[2,5,8,10]$. Thus, the association with other clinical and

Received on 15 September 2006; revised 23 November 2006.

Address for correspondence: Dr. Denise Duprat Neves. Avenida das Américas 1981, 33, Barra da Tijuca, Rio de Janeiro, Brazil. Zip code: 22631-000. E-mail: deduprat@gmail.com. Phone: 552124392522. Fax: 552125904640.

The Brazilian Journal of Infectious Diseases 2007;11(1):83-88. (C) 2007 by The Brazilian Journal of Infectious Diseases and Contexto Publishing. All rights reserved. laboratorial criteria has been suggested to improve test accuracy, increasing the specificity [1,2,7,10-12].

Our main purpose was to test the accuracy of clinical and laboratorial variables, in a predictive model, to the diagnosis of TB pleural effusion in an area of high prevalence of this disease.

\section{Materials and Methods}

This is a consecutive case series study, including all patients who entered a single protocol to investigate the cause of pleural effusion, from January 1997 to December 2001, in University Hospital Gaffrée \& Guinle, Rio de Janeiro, Brazil. Patients without ADA measure or no final diagnosis were excluded.

A diagnosis of TB was made when, at least, one of the following was present: Mycobacterium tuberculosis isolated from pleural fluid or tissue; presence of caseating granuloma in pleural biopsy or a noncaseating granuloma in pleura associated with positive smear for acid-fast bacilli or culture in other specimens, mainly sputum. The control group, without tuberculosis (NTB), included patients with transudates [13] and exudates due to malignancy, infection origin, besides others causes of pleural effusion as defined elsewhere [4].

Patients were interviewed to obtain their clinical history (duration of disease, symptoms and signs, co-morbidity). Patient's chest radiological features were evaluated by a pulmonologist. The routine analysis of the pleural fluid included: protein, lactate dehydrogenase, ADA, total and differential cell count, besides cytological exam. Histopathological exam was done when indicated.

Laboratorial exams were done by a technician blind to clinical data and according to current standards. ADA measurements were done in duplicate, by the method of Giusti.[14] Within one hour after thoracentesis, the sample, without anticoagulant, was centrifuged and the supernatant was aliquoted and stored at $20^{\circ} \mathrm{C}$ negative, until the day of the assay. The reading was done against controls for the reagents, substrates and sample. 
Variables studied were selected by the following criteria: recognition of some discriminatory power for the differential diagnosis of the pleural TB and the readiness and reliability of the collected information.

The best cut off value, for continuous variables, was selected through receiver operating characteristic (ROC) curve. Frequencies were compared by the chi-square $\left(\chi^{2}\right)$ statistic. All p values were two-sided, and statistical significance was set at 0.05 . The ability of each variable to correctly classify patients as TB or NTB was evaluated using odds ratios and the diagnostic accuracy of each variable was calculated according to standard methods [15] and previous published [16].

Variables found to be significant were included in a multivariate analysis through logistic regression. Collinearity was assessed using correlation coefficient. Variables were eliminated, one by one, in a backwards fashion, after being tested for interaction. The Hosmer-Lemeshow test was used to assess the fit of the logistic regression model. The sensitivity and specificity values and the area under curve (AUC) ROC were calculated to evaluate the models' final performance. Analysis was done using computer software: EPI-INFO 2000, World Health Organization, Geneva, Switzerland and MedCalc, from MedCalc Software, Belgium.

The local ethical committee approved the study and patients signed a written consent. There were no competing interests or financial support. Some results from this study have been previously reported in abstracts form [17].

\section{Results}

During recruitment period, 294 patients underwent thoracentesis; 79 cases were excluded due to inadequate specimen collection or inappropriate handling for ADA measurements (45 cases) and no conclusive final diagnosis (34 cases). Of the 215 remaining patients, 104 constituted the TB group, with a prevalence of 48\%, and 111 the NTB group. This included: 41 malignancies, with 7 lymphomas and 34 metastasis; 29 transudates; 28 parapneumonic effusions, being 12 simple and 16 complicated (14 empyema), and 13 other causes (3 secondary to systemic lupus erythematosus, 2 pulmonary embolization, 2 pancreatic disease, and the following with one case each: hemothorax, chylothorax, Dressler syndrome, endometriosis, secondary to chronic renal disease and hepatic disease).

Acid fast staining in pleural fluid was negative in all cases. Cultures were positive in less then $10 \%$, for pleural fluid, and $58 \%$, for the pleural biopsy specimens; a granuloma was detected in the pleural tissue in 95\% of confirmed TB cases. The mean age in patients with TB (mean=33.76; $\mathrm{SD}=13.96$ years old) was significant lower $(\mathrm{p}<0.0001)$ than in NTB group (mean=49.29; $\mathrm{SD}=18.01$ years old). In the TB group, 76 were men and in NTB group 60 patients $(\mathrm{p}=0.006)$.

The individual discriminating power of the analyzed variables is shown in Table1. Male was significantly more frequent in the TB group. The radiological patterns were similar in the two groups. ADA, cell type, protein and age were of greater discriminating power for the differential diagnosis, with an area under the ROC curve greater than 0.7 (not shown) and odds ratio more than 5 . The lactate dehydrogenase, total leukocytes and duration of disease had lower discriminating power, but were capable to differentiating the TB from the NTB group. Symptoms analysis did not show significant differences between TB and NTB patients.

The following variables were excluded from the multivariate analysis: percentile of polymorphonuclear, because it was highly correlated with the lymphocytes ( $r=-0.98)$; and radiological features which did not have statistical significance to discriminate the two groups.

Table 2 shows the predictive power and a summary of the variable elimination process based on the significance. The three equations refer to: model 1 - all selected variables; model 2 - excluding no significant variables, which is the model with best accuracy; model 3-variables with best accuracy in both, bivariate and multivariate, plus supported by the literature.

In Figure1 was displayed the ROC curves and in Figure 2 the dot plot diagram for ADA and the two models expressing the probability of tuberculous pleural effusion after applying the equation below with three and five variables in each case, respectively:

$\mathrm{Y}=0.0642 * \mathrm{ADA}+0.1028 * \mathrm{LYMPH}+0.6911 * \mathrm{PROT}+(-14.498)$, or

$\mathrm{Y}=0.1226 * \mathrm{ADA}+0.1088 * \mathrm{LYMPH}+2.0962 * \mathrm{PROT}+(-$ $0.0519 *$ Duration $)+(-0.0010 *$ LEUCO $)+(-19.1002)$, and then, calculating the probability of tuberculosis by applying: Probability of TB $[\mathrm{p}(\mathrm{Y}=1)]=1 /\left(1+\mathrm{exp}^{-\mathrm{Y}}\right)$.

\section{Discussion}

The identification of Mycobacterium tuberculosis in any specimen is sufficient for the diagnosis of tuberculosis, but in pleural tuberculosis this is not frequent. Due to the complexity of any disease, the presence or absence of a single feature can rarely determine the correct diagnosis. Hence, we performed a multivariate analysis including all variables that was capable to discriminate TB from NTB pleurisy, in a bivariate analysis.

The best predictive model of TB pleural effusion included five variables, as follows: ADA (U/L), lymphocytes (\%), protein (g/dL), duration of disease (days) and white cell count (cells $/ \mathrm{mm}^{3}$ ). This function showed sensitivity and specificity of more than 95\%, considering the cut off above 0.7 of probability of TB presence. The model that includes the three first variables may have a similar performance in clinical practice, with less calculations and utilization of more steady variables. The performance of some variables in the model is consistent with the results of individual analysis in discriminating TB from NTB described in other studies [1,2,7,10,18,19].

DLH did not prove usefulness in the multivariate analysis, being excluded, despite its utility in identifying parapneumonic complications and in discriminating transudate from exsudate. 
Table 1. Individual performance of each variable in discriminate of TB from NTB

\begin{tabular}{|c|c|c|c|c|c|c|c|}
\hline Variable & $\mathbf{N}$ & Cut-off & $X^{2}(p)$ & $\begin{array}{c}\text { OR } \\
95 \% \mathrm{CI}\end{array}$ & Accuracy & $\begin{array}{c}\text { Sens } \\
95 \% \text { CI }\end{array}$ & $\begin{array}{c}\text { Spec } \\
95 \% \text { CI }\end{array}$ \\
\hline Sex & 215 & masculine & 0.006 & $\begin{array}{c}2.31 \\
1.3-4.3\end{array}$ & 59.1 & $\begin{array}{c}73.1 \\
66-80\end{array}$ & $\begin{array}{c}45.9 \\
39-52\end{array}$ \\
\hline XR - local & 215 & unilateral & 0.140 & $\begin{array}{c}2.75 \\
0.8-10.6\end{array}$ & 46.5 & $\begin{array}{c}96.2 \\
92-99\end{array}$ & $\begin{array}{c}9.9 \\
3-12\end{array}$ \\
\hline XR - side & 215 & right & 0.386 & $\begin{array}{c}0.75 \\
0.4-1.4\end{array}$ & 51.6 & $\begin{array}{c}57.0 \\
49-64\end{array}$ & $\begin{array}{c}36.0 \\
29-43\end{array}$ \\
\hline XR - volume & 215 & $<2 / 3$ & 0.780 & $\begin{array}{c}1.14 \\
0.6-2.1\end{array}$ & 50.7 & $\begin{array}{c}71.2 \\
64-78\end{array}$ & $\begin{array}{c}31.5 \\
25-38\end{array}$ \\
\hline XR - lesion & 208 & no & 0.070 & $\begin{array}{c}1.93 \\
0.9-3.9\end{array}$ & 55.3 & $\begin{array}{c}82.4 \\
76-88\end{array}$ & $\begin{array}{c}29.2 \\
23-35\end{array}$ \\
\hline Age & 215 & $<$ 45years & 0.000 & $\begin{array}{c}6.90 \\
3.6-13.5\end{array}$ & 71.2 & $\begin{array}{c}80.8 \\
74-87\end{array}$ & $\begin{array}{c}62.2 \\
56-68\end{array}$ \\
\hline Duration & 211 & $<45$ days & 0.005 & $\begin{array}{c}2.69 \\
1.3-5.5\end{array}$ & 57.8 & $\begin{array}{c}84.2 \\
78-90\end{array}$ & $\begin{array}{c}33.6 \\
28-39\end{array}$ \\
\hline Protein & 214 & $>4.1 \mathrm{mg} / \mathrm{dL}$ & 0.000 & $\begin{array}{c}7.66 \\
3.8-15.8\end{array}$ & 70.5 & $\begin{array}{c}85.6 \\
79-91\end{array}$ & $\begin{array}{c}56.4 \\
50-61\end{array}$ \\
\hline DLH & 193 & > 298U/L & 0.001 & $\begin{array}{c}2.99 \\
1.6-5.8\end{array}$ & 62.2 & $\begin{array}{c}74.2 \\
67-81\end{array}$ & $\begin{array}{c}51.0 \\
44-57\end{array}$ \\
\hline Leukocytes & 194 & $\begin{array}{c}<6000 \\
\mathrm{cel} / \mathrm{mm}^{3}\end{array}$ & 0.001 & $\begin{array}{c}10.17 \\
2.2-65.2\end{array}$ & 55.8 & $\begin{array}{c}97.9 \\
93-99\end{array}$ & $\begin{array}{c}18.3 \\
14-20\end{array}$ \\
\hline Lymphocytes & 212 & $>81 \%$ & 0.000 & $\begin{array}{c}10.39 \\
4.9-22.6\end{array}$ & 72.6 & $\begin{array}{c}88.4 \\
82-93\end{array}$ & $\begin{array}{c}57.8 \\
52-62\end{array}$ \\
\hline $\mathrm{ADA}$ & 215 & > 39U/L & 0.000 & $\begin{array}{c}95.87 \\
31-310\end{array}$ & 88.8 & $\begin{array}{c}95.2 \\
90-98\end{array}$ & $\begin{array}{c}82.9 \\
78-86\end{array}$ \\
\hline
\end{tabular}

$\mathrm{N}=$ number of cases, $\mathrm{OR}=$ odds ratio, $\mathrm{XR}=\mathrm{X}$ rays, $\mathrm{DLH}=$ lactate dehydrogenase, $\mathrm{ADA}=$ adenosine deaminase, $\mathrm{AUC}$ ROC= area under ROC, Sens= sensibility, Spec= specificity. Cutoff value for continuous variable were calculated with ROC curve.

Table 2. Regression coefficients and significance values for three models used to differentiate TB from NTB pleural effusions

\begin{tabular}{|c|c|c|c|c|c|c|}
\hline & \multicolumn{2}{|c|}{ Model 1* } & \multicolumn{2}{|c|}{ Model 2* } & \multicolumn{2}{|c|}{ Model 3* } \\
\hline & $\mathbf{p}$ & Coef & $\mathbf{p}$ & Coef & $\mathbf{p}$ & Coef \\
\hline Sex & 0.865 & -0.2054 & & & & \\
\hline Age & 0.776 & -0.0077 & & & & \\
\hline Duration & 0.016 & -0.0566 & 0.0028 & -0.0519 & & \\
\hline Protein & 0.001 & 2.5618 & 0.0001 & 2.0962 & 0.0045 & 0.6911 \\
\hline $\mathrm{DLH}$ & 0.059 & 0.0009 & & & & \\
\hline Leukocytes & 0.001 & -0.0011 & 0.0002 & -0.0010 & & \\
\hline Lymphocyte & 0.002 & 0.1210 & 0.0003 & 0.1088 & 0.0000 & 0.1028 \\
\hline ADA & 0.000 & 0.1477 & 0.0000 & 0.1226 & 0.0000 & 0.0642 \\
\hline Constant & 0.0005 & -22.4635 & 0.0000 & -19.1002 & 0.0000 & -14.498 \\
\hline p value & & & \multicolumn{2}{|c|}{$\mathrm{p}>0.05$} & \multicolumn{2}{|c|}{$\mathrm{p}<0.05$} \\
\hline
\end{tabular}

*Model 1: all selected variables; Model 2: excluding non statistical significant variables; Model 3: variables with best accuracy in both bivariate and multivariate analyses. DLH=lactate dehydrogenase, ADA=adenosine desaminase. p value by Hosmer-Lemeshow test comparing model 2 and 3 with the first one.

Age has been useful in many predictive models for pulmonary TB, especially in areas with high prevalence,[2,18,20,21,22] but was useless in our final model.

The best accuracy was observed for ADA. Choosing the cut off point greater than $39 \mathrm{U} / \mathrm{L}$, this variable yields a sensitivity of $95.2 \%$ (95\%CI 90 to 98 ) and a specificity of $82.9 \%$ (95\%CI 78 to 86 ), which is similar to other reports
$[2,5,6,7,8,9,23]$. The specificity was even greater (93\%) when considering the nonempyematic effusions.

The presence of lymphocytic pleural effusion has been useful in the diagnosis of TB pleural effusion. Its combination with ADA has been evaluated with the purpose of increasing specificity, probably because it is useful in excluding the empyema, one of the most frequent causes of ADA's false 
Figure 1. Compare ROC curve for ADA, model with 3 and 5 variables.
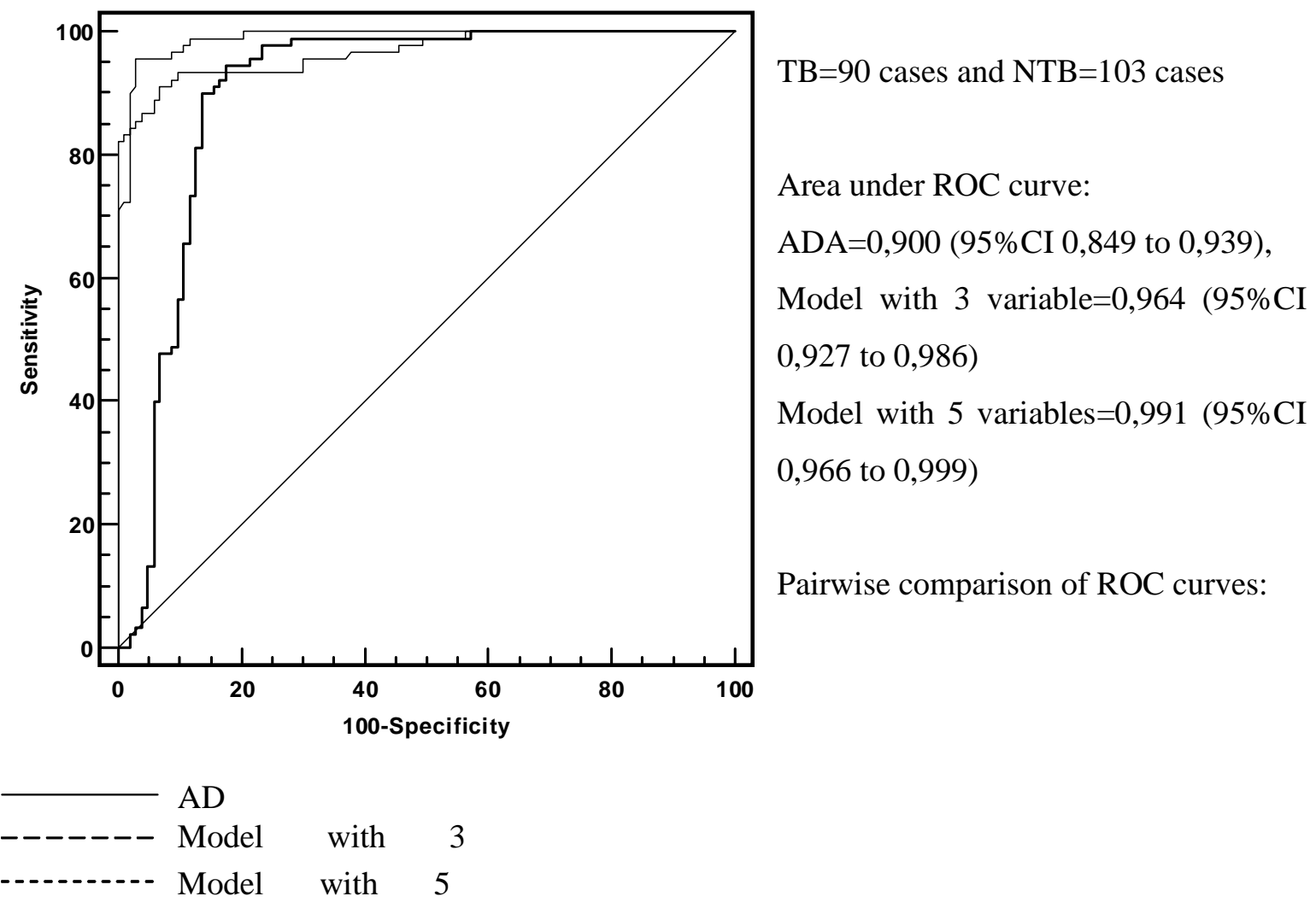

positive $[1,5,10,11,23]$. In general, this was the variable with best efficiency after ADA, individually or associated with other variables.

Protein performed similar to other reports [2,7,9]. Due to the scale, the protein's adjusted OR is greater than ADA and lymphocytes but its contribution to the diagnosis is smaller. It is possible that it contributions is based on the exclusion of the lymphoma, another cause of ADA false positive.

Duration of disease and total number of leukocytes were important to discriminate TB from NTB, but only in multiple logistic regression. The total number of leukocytes was able to distinguish TB from transudates, with lower cell counts, and from parapneumonic effusions, with higher cell counts $[4,13]$. Due to its great variability within the same disease, its reproducibility could be poor. Besides, its performance may be poor in patients with co-infection with TB and HIV, in whom leucocytes counts is lower [2,9].

Infectious pleural effusions, like parapneumonic effusions and tuberculosis, have smaller duration of disease, especially when compared to neoplasic effusions. However, this information may not be quite accurate to be consisting in the models, because it depends on the patient's perception and medical interpretation. The beginning of the symptoms is not always coincident with the formation of the pleural effusion, the symptoms may not be valued because they are mild and therefore neglectful, or they are associated with other complaints of the baseline disease.

Few authors had studied the diagnosis of TB pleural effusion using multivariate analysis and the results have some controversies. Carrion-Valero et al. [20] assessed the value of discriminate analysis as a method of optimizing the diagnosis of pleural TB but they did not included ADA in their model. A clinical score for differential diagnosis between TB and malignant pleural effusions, with retrospective data, was evaluated using multivariate analysis and the authors derived two models, with and without ADA [24]. Ghanei et al. [19] examined a statistical method by combining the diagnostic efficiency of ADA, pleural fluid protein, lactate dehydrogenase and cellular components to the diagnosis of pleural tuberculosis. Therefore, its seems to be a new and promising field of investigation.

In areas of high prevalence of TB, more than $50 \%$ of pleural effusion of indeterminate cause is due this disease. The model should be viewed as a rapid and accurate method of suggesting the diagnosis of TB pleural effusion when other exams were negative or not possible to be done. In many of the confirmed TB cases granulomata were found, and this underlines the importance of pleural biopsies in the diagnosis of pleural TB. So the model should not be used as an alternative to biopsy and culture, but rather as an adjuvant tool for the 
Figure 2. Dot plot diagram for ADA (in A), predicted model with 3 variables (in B) and predicted model with 5 variables (in C). In all are shown the best cut off value and the sensitivity and specificity considering this point.
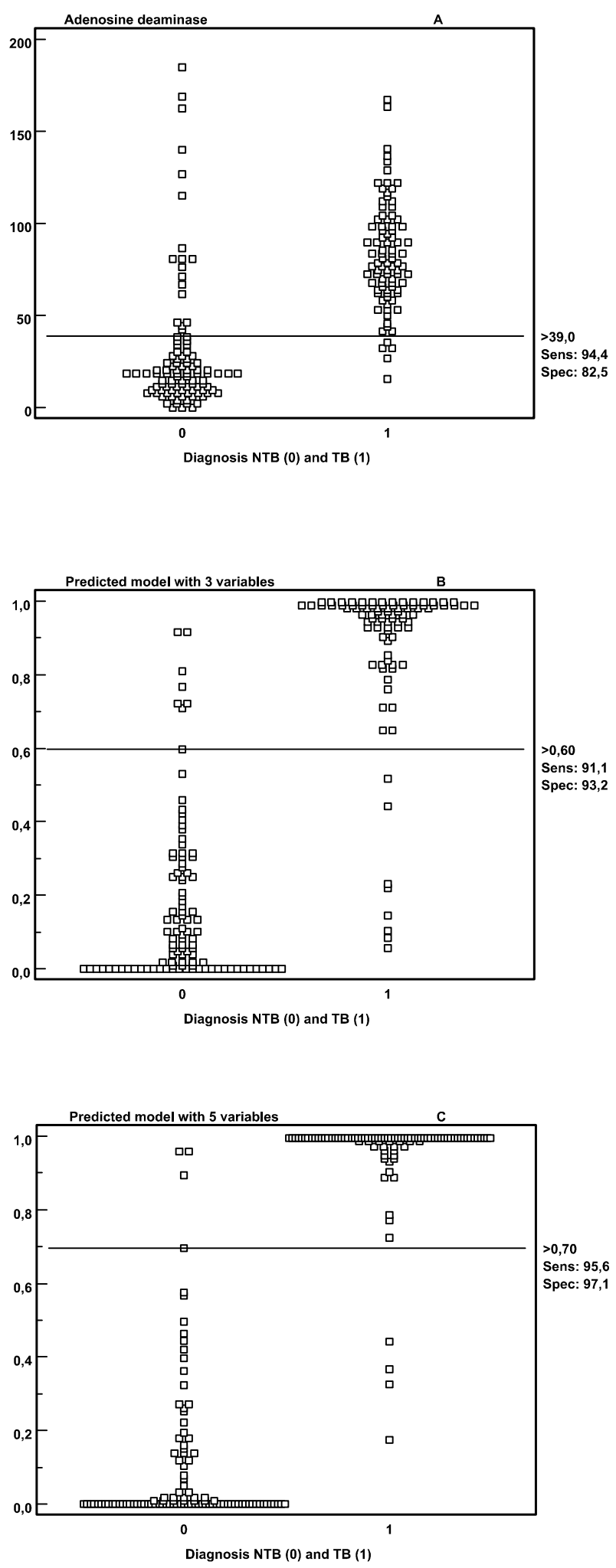

criteria of "probable TB pleural effusion", optimizing the utilization of more expensive or invasive tests, allowing earlier diagnosis and preventing the unnecessary tuberculosis chemotherapy. The advantage of the predictive model we developed is that it included variables that are readily available from routine investigation of pleural effusion.

How the clinician is going to use the results of this study in practice will depends on its confirmation in the population where it will be applied. Although our and others studies may have internal validity, any predictive model should be tested and the accuracy of the model should be confirmed prospectively. Then, the probability of TB for each patient according to the equation may be transform in a score, so that it will be easier to use by clinicians on a routine basis. We recommend the test with exclusion of empyema pleural effusion, what may improve the performance of the model with lesser number of variables.

\section{References}

1. Oliveira H.G., Rossatto E.R., Prolla J.C. Pleural fluid adenosine deaminase and lymphocyte proportion: clinical usefulness in the diagnosis of tuberculosis. Cytopathology 1994;5(1):27-32.

2. Melo F.A.F., Afiune J.B., Santos M.L., Castelo Filho A. Diagnóstico da tuberculose pleural pela ADA, isolada ou combinada a outras variáveis, inclusive em HIV-positivos. Folha Médica 2000;119(3):9-21.

3. Seibert A.F., Haynes Jr. J., Middleton R., Bas J.B. Tuberculous pleural effusion - twenty-year experience. Chest 1991;99:883-6.

4. Light R.W. Pleural Diseases. 3rd ed. Philadelphia: Lea \& Febiger; 1995.

5. Ena J., Vallis V., Oteyza C.P., Salamanca R.E. Utilidad y limitaciones de la adenosina desaminasa en el diagnóstico de la pleuresía tuberculosa. Estudo metaanalítico. Med Clin (Barc) 1990;95:333-5.

6. Aoki Y., Katoh O., Nakanishi Y., et al. A comparison study of IFN-gamma, ADA, and CA125 as the diagnostic parameters in tuberculous pleuritis. Respir-Med 1994;88(2):139-43.

7. Kim Y.C., Pak K.O., Bom H.S., et al. Combining ADA, protein and IFN-gamma best allow a discrimination between tuberculous and malignant pleural effusion [abstract]. Korean J Intern Med 1997;12(2):225-31.

8. Blake J., Berman P. The use of adenosine deaminase assays in the diagnosis of tuberculosis. S Afr Med J 1982;62(1):19-21.

9. Riantawan P., Chaowalit P., Wongsangiem M., Rojanaraweewong P. Diagnostic value of pleural fluid adenosina deaminase in tuberculous pleuritis with reference to HIV coinfection and bayesian analysis. Chest 1999;116:97-103.

10. Burgess L.J., Maritz F.J., Roux I., Taljaard J.J.F. Combined use of pleural adenosine deaminase with lymphocyte/neutrophil ratio. Increased specificity for the diagnosis of tuberculous pleuritis. Chest 1996;109(2):414-9.

11. Lee Y.C.G., Rogers J., Rodriguez R.M., et al. Adenosine deaminase levels in nontuberculous lymphocitic pleural effusions. Chest 2001;120(2):356-61.

12. Ferrer J.S., Munoz X.G., Orriols R.M., et al. Evolution of idiopathic pleural effusion: a prospective, long-term follow-up study. Chest 1996;109:1508-13.

13. Light R.W., Macgregor M.I., Luchsinger P.C., Ball W.C. Jr. Pleural effusions: The diagnosis separation of transudates and exsudates. Ann Int Med 1972;77(4):507-13.

14. Giusti G. Adenosine deaminase. In: Bergmeyer H.U., editor. Methods of Enzymatic Analysis. New York: Academic Press; 1974. p. 1093-9. 
15. Bossuyt P.M., Reitsma J.B., Bruns D.E., et al. Towards complete and accurate reporting of studies of diagnostic accuracy: the STARD initiative. BMJ 2003;326(7379):41-44.

16. Neves D.D., Dias R.M., Cunha A.J.L.A., Chibante A.M.S. Rendimento de variáveis clínicas, radiológicas e laboratoriais para o diagnóstico da tuberculose pleural. J Bras Pneumol 2004;30(4):409-16.

17. Neves D.D., Dias R.M., Cunha A.J.L.A. Preditive model for diagnosis of tuberculous pleural effusion. Am J Respir Crit Care Med 2003;167(7):A902.

18. Valdes L., Alvarez D., San José E., et al. Value of adenosine deaminase in the diagnosis of tuberculous pleural effusions in young patients in a region of high prevalence of tuberculosis. Thorax 1995;50:600-3.

19. Ghanei M., Aslani J., Bahrami H., Adhami H. Simple Method for Rapid Diagnosis of Tuberculosis Pleuritis: A Statistical Approach. Asian Cardiovasc Thorac Ann 2004;12(1):23-9.
20. Carrion-Valero F., Perpiña-Tordera M. Screening of tuberculous pleural effusion by discriminant analysis. Int J Tuberc Lung Dis 2001;5(7):673-9.

21. Roper W.H., Waring J.J. Primary serofibrinous pleural effusion in military personnel. Am Rev Tuberc 1955;71:616-35.

22. Villegas M.V., Labrada L.A., Saravia N.G. Evaluation of polymerase chain reaction, adenosine deaminase and interferon- $\gamma$ in pleural fluid for the diferential diagnosis of pleural tuberculosis. Chest 2000;118(5):1355-64.

23. Ocaña I., Martinez-Vázquez J.M., Ribera E., et al. Adenosine deaminase activity in the diagnosis of lymphocytic pleural effusions of tuberculous, neoplastic and lymphomatous origin. Tubercle 1986;67:141-5.

24. Porcel J.M., Vives M. Differentiating tuberculous from malignant pleural effusions: a scoring model. Med Sci Monit 2003;9(5):CR227-32. 